\title{
Mixofibroma odontogénico, reporte de un caso con seguimiento a cinco años.
}

\author{
Odontogenic myxofibroma, case report with 5 years of follow-up.
}

\author{
Yamely Ruiz-Vázquez, ${ }^{*}$ Ricardo Rahuel Espino-Tejeda, ${ }^{\ddagger}$ Beatriz Catalina Aldape-Barrios ${ }^{\S}$
}

\section{RESUMEN}

El mixoma odontogénico es una neoplasia de origen mesenquimal, con crecimiento lento pero localmente invasivo y agresivo, que cuenta con un aspecto histológico característico. Radiográficamente, la mayoría de las lesiones tienen un aspecto radiolúcido multilocular, descrito en la literatura como «panal de abejas» o «burbujas de jabón», que ocasionan desplazamiento dental y expansión corticales. En el ámbito histopatológico, se caracteriza por células estelares o fusiformes con prolongaciones citoplasmáticas, en una sustancia mucoide o mixoide con presencia o no de colágena, cuando contiene grandes cantidades de tejido fibroso celular maduro se le llama mixofibroma. Se presenta un caso clínico de hombre de 38 años, que acude al Hospital General de Uruapan, Michoacán con aumento de volumen en región maxilar derecha; se toma biopsia incisional y se emite el diagnóstico histopatológico de mixofibroma odontogénico, $33.6 \%$ de los casos se presenta en el maxilar, con una distribución uniforme por todas las áreas llegando a erosionar el seno maxilar. Tiene una tasa de recurrencia de 10 a $33 \%$, y el tratamiento ideal es la resección en bloque con márgenes de seguridad.

Palabras clave: Mixofibroma, mixoma, odontogénico, resección en bloque, biopsia.

\section{ABSTRACT}

Odontogenic myxoma is a slow-growing locally invasive intraosseous lesion with defined microscopic appearance. Radiographic appearance, is described as multilocular radiolucent lesion, which is reported in scientific literature as «honeycomb» or «soap bubbles» appearance most of the time; it exhibits dental displacement and cortical expansion. Histopathologically, stellate and spindle cells with cytoplasmic processes are observed in a mucoid or myxoid substance, with or without collagen; when it contains large amounts of mature cellular fibrous tissue, it is called myxofibroma. The present article presents the case of a 38 year old male, who attended the General Hospital in Uruapan, Michoacán due to an increased volume in the right maxillary region. Incisional biopsy was performed and the histopathological diagnosis of odontogenic myxofibroma was emitted. $33.6 \%$ of cases occur in the maxilla with a uniform distribution throughout the areas, and can erode the maxillary sinus. It has a recurrence rate of 10 to $33 \%$, therefore ideal treatment is bloc resection leaving safety margins.

Keywords: Myxofibroma, myxoma, odontogenic, bloc resection, biopsy.

\section{INTRODUCCIÓN}

$\mathrm{E}$ n 1863, Rudolf Virchow introduce el término mixoma para designar a las lesiones mesenquimatosas que histológicamente semejan a la sustancia mucoide del cordón umbilical. ${ }^{1}$ Pero es hasta 1947 cuando Thoma KH y Goldman HM renombran como mixoma odontogénico a la lesión con esas características pero que aparece en la región de maxilar y mandíbula. ${ }^{2,3}$

El mixoma es una neoplasia que deriva del ectomesénquima odontogénico, debido a que microscópicamente se parece a la papila dental de un diente en desarrollo. ${ }^{4}$ Es el tercer tumor odontogénico más común, seguido del odontoma y ameloblastoma, con una prevalencia de $2-5 \% .{ }^{5}$

\footnotetext{
* Maestra en Patología y Medicina Bucal, práctica privada. México.

‡ Cirujano Maxilofacial, Hospital General «Dr. Pedro Daniel Martínez». Uruapan, Michoacán. México.

$\S$ Especialista y Maestra en Patología Bucal, Profesor de tiempo completo en la Facultad de Odontología, UNAM. México.

Recibido: 04 de agosto de 2019. Aceptado: 19 de julio de 2021.
}

Citar como: Ruiz-Vázquez Y, Espino-Tejeda RR, Aldape-Barrios BC. Mixofibroma odontogénico, reporte de un caso con seguimiento a cinco años. Rev ADM. 2021; 78 (4): 235-239. https://dx.doi.org/10.35366/101079 
Se define como una lesión benigna intraósea, de crecimiento lento, localmente invasiva y agresiva. Setenta y cinco por ciento de los casos están entre la tercera y cuarta década de la vida, con un promedio de edad de 30 años.., 6 Algunos autores reportan una ligera predisposición por el sexo masculino, con una razón 1.5:1 respecto a las mujeres, pero la mayoría reporta que no existe predilección por el sexo. ${ }^{4}$ Se presenta con mayor incidencia en mandíbula (66.4\%), principalmente en la región molar, cerca de la rama mandibular e incluso el cóndilo; y únicamente el $33.6 \%$ se presenta en el maxilar. ${ }^{7,8}$

El mixoma odontogénico se presenta como un aumento de volumen con asimetría facial, asintomáticos, que en la región intrabucal puede estar ulcerado y ocasionar desplazamiento dental y expansión de corticales hasta en un $74 \%$ de los casos. ${ }^{6,9}$ La reabsorción de raíces se describe en un $9.5 \%$ y únicamente un $5 \%$ de los casos reportados están asociados a dientes no erupcionados, y cuando está involucrado el seno maxilar puede ocasionar obstrucción nasal y exoftalmos. ${ }^{6}$

Radiográficamente se describe como una lesión radiolúcida multilocular, con un patrón en «panal de abejas», «raqueta de tenis» o «burbujas de jabón» en lesiones grandes; aunque puede aparecer como una imagen unilocular hasta en un $36 \%$, principalmente en lesiones menores a 4 centímetros. 5,7,10,11 Los diagnósticos diferenciales imagenológicos incluyen a otras lesiones radiolúcidas con el mismo patrón, como el ameloblastoma, el queratoquiste odontogénico o la lesión central de células gigantes. Sin embargo, las lesiones pequeñas son de crecimiento asintomático y son descubiertas como hallazgos imagenológicos..$^{9,11}$

En el ámbito macroscópico, la consistencia de la neoplasia es blanda, o bien, puede ser firme en las neoplasias con mayor presencia de colágena, el color va de blanco a blanco grisáceo, y al realizar un corte, la superficie interna presenta un aspecto gelatinoso de aspecto mucoide. ${ }^{5,10,12}$
En la histopatología se observan células estelares, fusiformes y redondas con prolongaciones citoplasmáticas que se anastomosan, dispuestas de forma irregular (en «araña»). Estas células se encuentran dispersas en un fondo de sustancia mixoide o mucoide, formada por glucosaminoglicanos, como el ácido hialurónico y que puede presentar grandes cantidades de colágena; cuando esto sucede, recibe el nombre de mixofibroma o fibromixoma. $5,7,10,13$ Los núcleos son de forma oval e hipercromáticos, y las mitosis son hallazgos poco frecuentes. En ocasiones se observan islas o cordones inactivos de epitelio odontogénico, y en la periferia se pueden observar zonas de material calcificado. Este tejido mixoide infiltra en los espacios trabeculares óseos, lo que puede explicar el porqué es difícil retirar la lesión.

\section{PRESENTACIÓN DEL CASO}

Se presenta un hombre de 38 años, que al interrogatorio niega cualquier antecedente patológico que se involucre con el diagnóstico del padecimiento actual. Acude a revisión por aumento de volumen en la región maxilar de lado derecho de un año de evolución aproximadamente, con curso asintomático; a la exploración clínica se observa el aumento de volumen similar al color de la mucosa adyacente. Refiere haber sido tratado con antibióticos durante un mes y medio, sin obtener respuesta favorable. Se realizó la reconstrucción en tercera dimensión como auxiliar de diagnóstico, donde fue posible apreciar la expansión y pérdida de la cortical ósea en el hueso maxilar de lado derecho causado por la presencia de la lesión, además de desplazamiento dental (Figura 1).

Asimismo, se hizo la toma de biopsia incisional de la lesión para llevar a cabo el estudio histopatológico. Macroscópicamente se recibieron múltiples fragmentos de tejido blando fijados en formalina, de superficie y forma
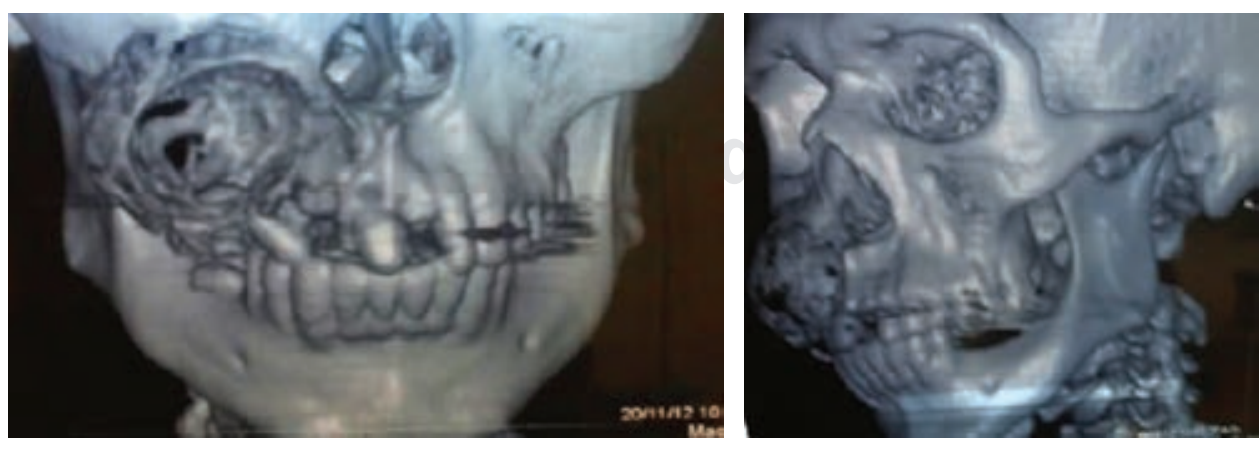

Figura 1:

Destrucción ósea en reconstrucción 3D. 
En hematoxilina y eosina se observan células fusiformes con prolongaciones citoplasmáticas en un estroma de tejido mixoide con zonas de abundantes haces de colágena.
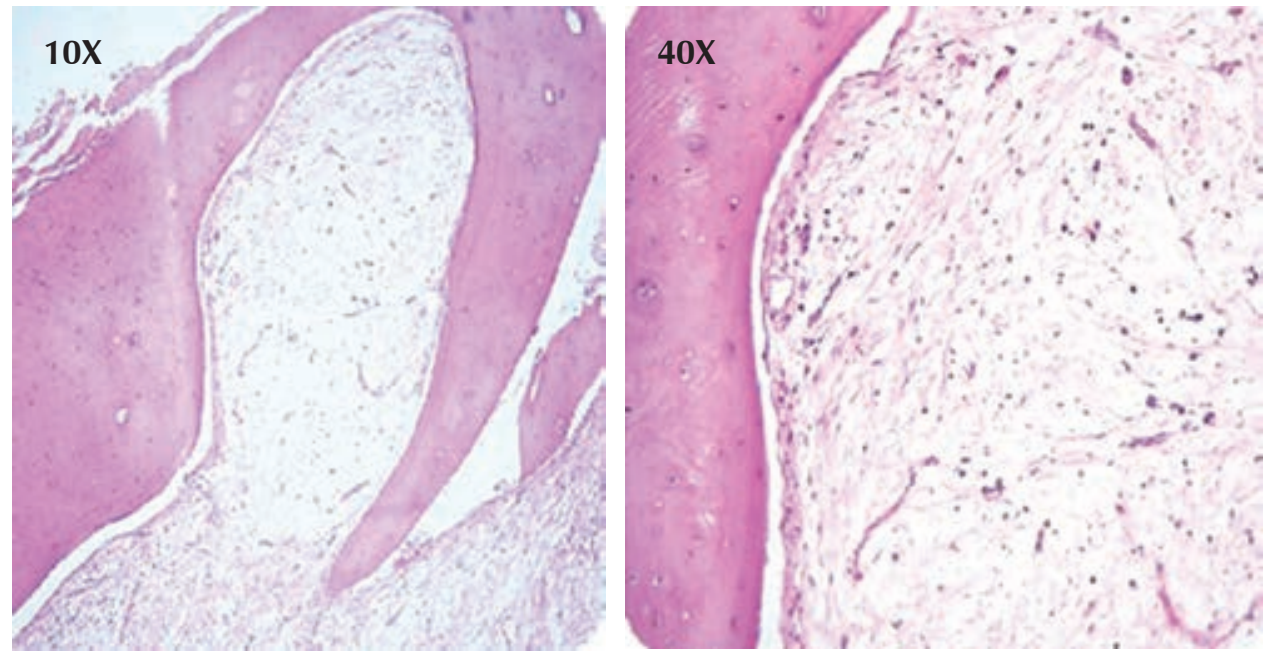

Figura 2:
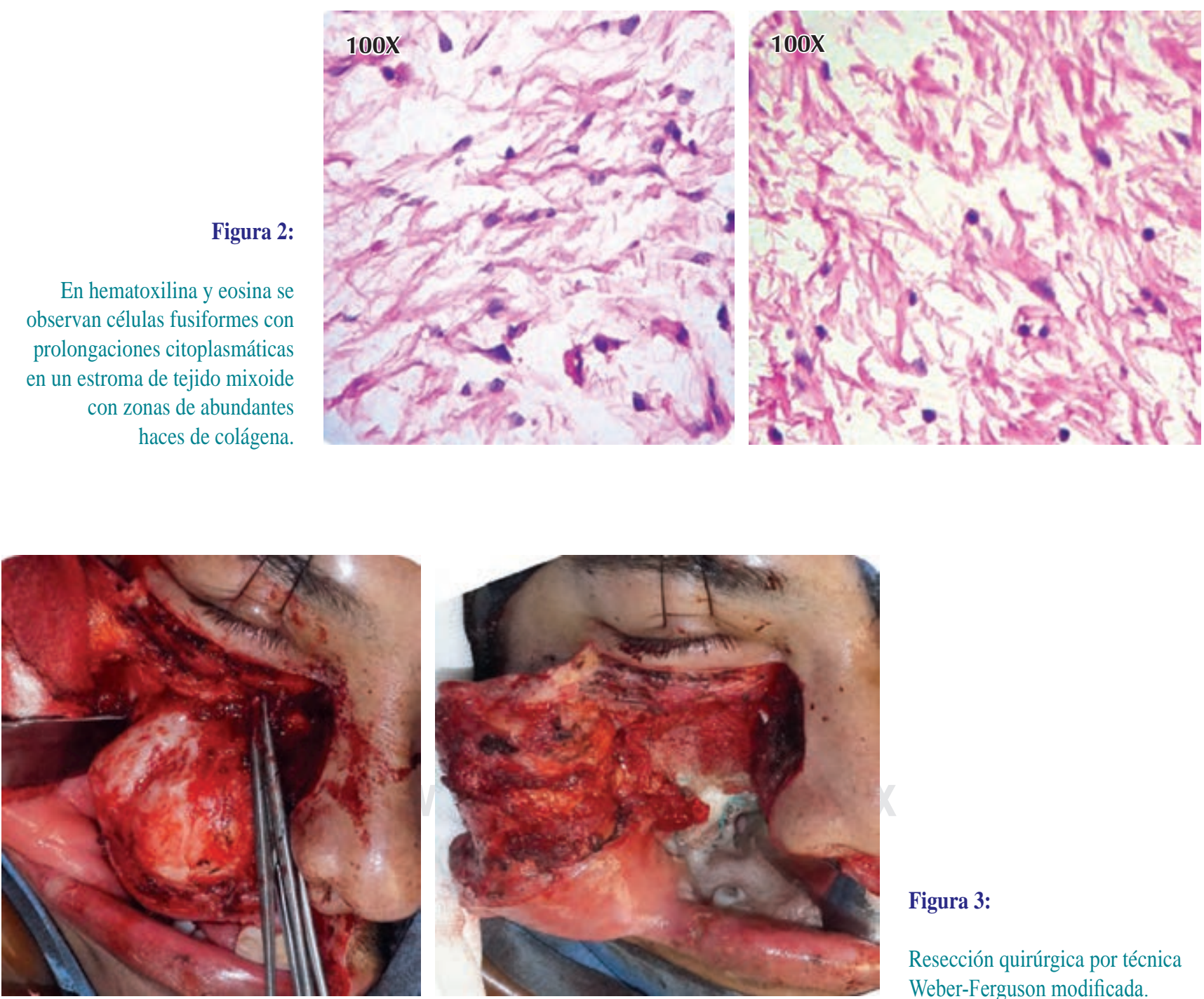

Figura 3:

Resección quirúrgica por técnica Weber-Ferguson modificada. 
irregular, de color café claro, de consistencia blanda, que en conjunto midieron $2.5 \times 2.0 \times 0.6 \mathrm{~cm}$. El estudio histopatológico mostró la presencia de células fusiformes y estelares con prolongaciones citoplasmáticas, dispuestas en un tejido conectivo laxo de aspecto mixoide con áreas evidentes de tejido conectivo fibroso denso bien vascularizado (Figura 2), se emitió el diagnóstico de mixofibroma odontogénico. Con los datos imagenológicos, clínicos, los estudios de laboratorio correspondientes y el resultado de la biopsia incisional, se programa la resección quirúrgica completa.

Se realizó la resección del tumor por medio de hemimaxilectomía con técnica quirúrgica de Weber-Ferguson modificada (Figura 3), la pieza quirúrgica se fijó en formol a 10\%, y se envió a su estudio histopatológico, en donde se confirmó el diagnóstico de mixofibroma odontogénico.

Después de la cirugía, el paciente fue rehabilitado con un obturador palatino de material metal-acrílico.
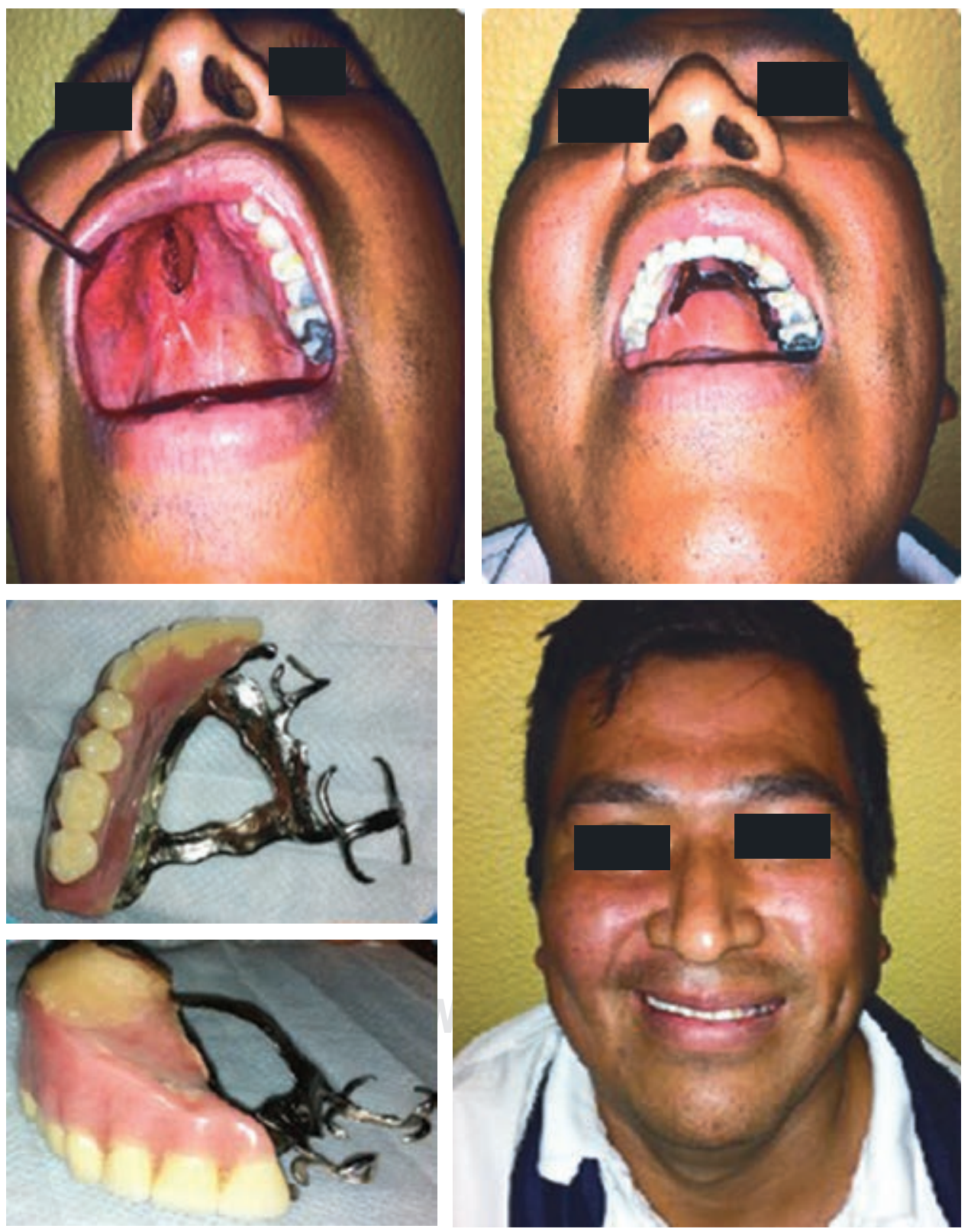

Figura 4:

Seguimiento a siete meses, paciente completamente rehabilitado con obturador maxilar. 
Se da seguimiento a la semana, dos semanas, uno, tres y siete meses, y de manera anual en donde se observa la adecuada cicatrización de las heridas quirúrgicas; sin embargo, hay comunicación oroantral, la cual es obturada con la prótesis que usa el paciente (Figura 4). Se observa pérdida del tono labial superior del lado de la lesión, y aún hay cierto grado de asimetría facial, aunque después de cinco años de seguimiento no se ha reportado recidiva.

\section{DISCUSIÓN}

Existen distintas clases de lesiones de aspecto mixoide, como el folículo hiperplásico, liposarcoma mixoide, condrosarcoma mixoide, fibroma condromixoide y el neurofibroma mixoide, que deben considerarse durante el diagnóstico histopatológico, en ocasiones tendrán que descartare con estudios de inmunohistoquímica, en especial en casos con presencia de mitosis y alta actividad celular. ${ }^{10,12,14}$

Si bien existen mixomas en tejidos blandos y otros huesos, el término mixoma odontogénico se debe a la localización en maxilar y mandíbula, por esto, aunque la presencia de epitelio odontogénico no es fundamental para su diagnóstico, cuando éstos se presentan, pueden llegar a mostrar zonas de hialinización rodeando las islas epiteliales. 5, 10,14

Es importante tomar en cuenta diagnósticos diferenciales imagenológicos como el ameloblastoma, la lesión central de células gigantes, quiste óseo aneurismático y el queratoquiste odontogénico, además de evaluar todas las características imagenológicas de la lesión, como expansión de corticales, desplazamiento y reabsorción dental. ${ }^{9}$

Se han reportado casos de mixomas odontogénicos periféricos, principalmente en encía, y casos reportados con asociación a esclerosis tuberosa. ${ }^{10}$

\section{CONCLUSIONES}

El mixoma odontogénico es una lesión de crecimiento lento, pero infiltrativa y agresiva, con un rango de recurrencia de $10-33 \%$, relacionada de manera directa con la terapia; en terapias conservadoras como curetaje, la recurrencia es mayor en comparación con la resección en bloque. El presente caso fue tratado con resección quirúrgica de la neoplasia con márgenes de seguridad, y después del seguimiento a cinco años no se ha observado recidiva.

\section{REFERENCIAS}

1. Thoma, Kurt H. Patología oral. Barcelona, España: Salvat Editores; 1973.

2. Simon EN, Merkx MA, Vuhahula E, Ngassapa D, Stoelinga PJ. Odontogenic myxoma: a clinicopathological study of 33 cases. Int J Oral Maxillofac Surg. 2004; 33 (4): 333-337.

3. Peltola J, Magnusson B, Happonen RP, Borrman H. Odontogenic myxoma--a radiographic study of 21 tumours. Br J Oral Maxillofac Surg. 1994; 32 (5): 298-302.

4. Neville BW. Oral and maxillofacial pathology. 4th ed. Canada: Elsevier; 2017. pp. 679-681.

5. El-Naggar AK, Chan JKC, Takata T, Grandis JR, Slootweg PJ. WHO classification of head and neck tumours. 4th ed. Lyon: IARC Press; 2017. pp. 266-267.

6. Kaffe I, Naor H, Buchner A. Clinical and radiological features of odontogenic myxoma of the jaws. Dentomaxillofac Radiol. 1997; 26 (5): 299-303.

7. Kumar N, Kohli M, Pandey S, Agarwal P. Odontogenic myxoma. J Maxillofac Oral Surg. 2014; 13 (2): 222-226.

8. Lahey E, Woo SB, Park HK. Odontogenic myxoma with diffuse calcifications: a case report and review of the literature. Head Neck Pathol. 2013; 7 (1): 97-102.

9. Zhang J, Wang H, He X, Niu Y, Li X. Radiographic examination of 41 cases of odontogenic myxomas on the basis of conventional radiographs. Dentomaxillofac Radiol. 2007; 36 (3): 160-167.

10. Reichart PA, Philipsen HP. Odontogenic tumors and allied lesions. London: Quintessence Publishing Co, Ltd; 2004.

11. Friedrich RE, Scheuer HA, Fuhrmann A, Zustin J, Assaf AT. Radiographic findings of odontogenic myxomas on conventional radiographs. Anticancer Res. 2012; 32 (5): 2173-2177.

12. Li TJ, Sun LS, Luo HY. Odontogenic myxoma: a clinicopathologic study of 25 cases. Arch Pathol Lab Med. 2006; 130 (12): 17991806.

13. Kawase-Koga Y, Saijo H, Hoshi K, Takato T, Mori Y. Surgical management of odontogenic myxoma: a case report and review of the literature. BMC Res Notes. 2014; 7: 214.

14. Noffke CE, Raubenheimer EJ, Chabikuli NJ, Bouckaert MM. Odontogenic myxoma: review of the literature and report of 30 cases from South Africa. Oral Surg Oral Med Oral Pathol Oral Radiol Endod. 2007; 104 (1): 101-109.

\section{Correspondencia: \\ Yamely Ruiz-Vázquez \\ E-mail: yaruva6@hotmail.com}

Conflicto de intereses: Los autores declaran no tener ningún conflicto de intereses.

Financiamiento: Ninguno. 